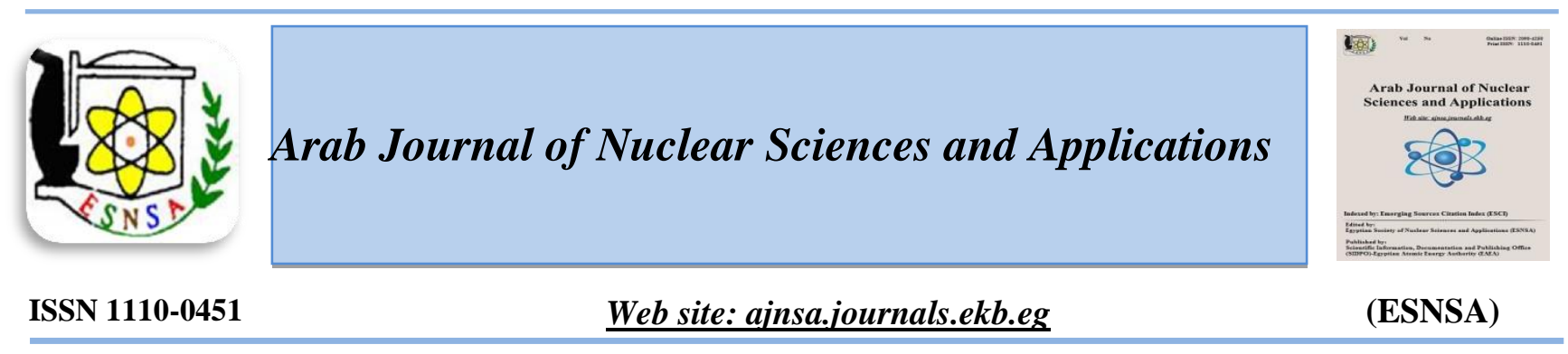

\title{
Dosimetric Evaluation of the Field-in-Field Technique for Large Breast Cancer Irradiation
}

\author{
${ }^{1}$ Mohammed Elywa, ${ }^{2}$ Shaimaa Elnabawy \\ ${ }^{1}$ Biophysics branch, Physics Department, Faculty of Science, Zagazig University, Egypt \\ ${ }^{2}$ Medical Physics Department, Meetghamr Oncology Center, Egypt
}

\begin{abstract}
Received $31^{\text {st }}$ May 2018 The aim of this work is using multi segmented conformal radiotherapy, field in field (FIF) for large Accepted $21^{\text {st }}$ July 2018 breast cancer treatment, the dose coverage of the planning target volume (PTV) and evaluation the radiation load on the organs at risk (OARs). Ten patients with large breasts of mean PTV was $1489 \pm$ $335 \mathrm{Cm}^{3}$ were included. Many parameters were used in the dose evaluations in the PTV OARsvolume including ipsi lateral lung, heart, and the contralateral breast, Dose homogeneity index (DHI) and conformity index (PITV). The FIF technique for all patients improved the homogeneity and conformity of the PTV, where the mean values of maximum dose of the PTV was $107 \%$, the volumes over the prescription dose in irradiated volumes received very low doses. For each dosimetry of the organs at risk, the FIF technique reduced the dose that these organs received. It could be concluded that the FIF technique provided a better dose distribution in the PTV, reduced the doses in the OARs and improved the homogeneity and conformity of the planning volume.
\end{abstract}

Keywords: linear accelerator (LINAC), FIF technique, Planning target volume, Dose homogeneity index, Breast cancer

\section{Introduction}

Most patients in the early-stage breast cancer have been subjected to the breast-conserving treatment consisting of a wide excision and post-operative radiotherapy. Post-operative radiotherapy reduces the risk of local recurrence and results in long-term survival similar to that obtained with mastectomy [1-3]. Post-operative breast tangential radiotherapy is a standard treatment for breast cancer radiotherapy. Women with large breasts are also more likely to have a little bit cosmetic outcome after radiotherapy relative to the women with smaller and medium sized breasts $[4,5]$.

In recent years, the field-in-field (FIF) technique has become a widely preferred method for administering tangential whole breast radiotherapy and several studies reported that this technique is useful in reducing hot regions as well as cold regions [6-14].

The optimal method for the FIF technique in the breast tangential radiotherapy has been determined [15]. The prone technique is the most commonly used for large breast as it offers the advantages of eliminating skin folds due to the gravitational effect of the hanging breast, therefore reducing the skin reaction. [5, 16 ]. The reduction of skin folds is beneficial to the patient since there is no buildup of the dose in the underlying tissue [5]. However, it has many disadvantages Namely, the prone device can be difficult for some patients to mount, depending on their level of physical capability while also being only moderately comfortable.

In this work, the authors used the FIF plan on a large breast size with supine position. An

Corresponding author: elywa2006@gmail.com

DOI: 10.21608 /ajnsa.3724.1093

(C) Scientific Information, Documentation and Publishing Office (SIDPO)-EAEA 
improved breast TP method has been prepared in an attempt to combine the advantages of threedimensional conformal radiation therapy (3DCRT) [17] and intensity-modulated radiation therapy (IMRT), with maintenance of the quality assurance, namely fast TP, short treatment time in 3D-CRT, improved dose homogeneity on the planning target volume (PTV), and reduced doses to the organs at risk (OARs) in IMRT. A radiotherapy plan, using many parameters such as conformity index and dose homogeneity index, has been simulated.

\section{Materials and methods \\ Patients}

Ten patients with early stage breast cancer were enrolled in this planning study. The mean volume of the breast was $1489 \pm 335 \mathrm{~cm}^{3}$ and the mean chest wall separation was $23.6 \mathrm{~cm}$, where the chest wall separation is the distance between the medial and lateral field borders at the level of the center [18]. Patients with lymph node metastasis or distant metastasis were excluded from the study. The patients were imaged with a CT scanner in treatment position (supine, the ipsi lateral arm up and head turned to the contralateral side). To maintain the treatment position, a breast board was fixed to the CT and treatment table. CT data were acquired with adjacent axial slice spacing of $5 \mathrm{~mm}$, covering the entire chest with normal free breathing. The data obtained from CT were transferred to the treatment planning system (TPS) (Prowess, 5.01). By using this planning system, the ipsi lateral lung, contralateral breast and heart were defined as the OARs. Planning target volume (PTV), heart, and contralateral breast were delineated by the same radiation oncologist. The dose calculation algorithm used was based on the pencil-beam convolution method. Six patients were generated using $6 \mathrm{MV} \mathrm{X}$-rays, four patient were generated using10 MV at Siemens PRIMUS and PRIMUS Plus . 82-leaf multi leaf collimator, two photon energies of $6 \mathrm{MV}$ and10MV and six electron energies (5Mev, $7 \mathrm{Mev}, 8 \mathrm{Mev}, 10 \mathrm{Mev}$, $12 \mathrm{Mev}$ and $14 \mathrm{Mev}$ ) have been applied using SoftwareVersionV8.0, Germany. The patients with used 10MV were with breast volume larger than $1300 \mathrm{~cm}^{3}$ and the separation was larger than 24 $\mathrm{cm}$. The prescription dose was 40 Gy in 15 fractions (266.6 Gy per fraction). The PTVs were restricted to $5 \mathrm{~mm}$ and $8 \mathrm{~mm}$ under the skin surface for $6 \mathrm{MV}, 10 \mathrm{MV}$ respectively, to exclude the buildup region from the PTVs.

\section{Field-in-Field radiotherapy}

All the treatment plans were created using the alternate subfields method ASM for the FIF technique and the starting point was the conventional two tangential plans. Then, additional multi leaf collimators MLC subfields were added. The medial fields were copied as the first subfield. Using MLCs for blocking the dose to the first subfield was $1 \%$ to $3 \%$ lower than the maximum dose on the beam's eye view. After estimation of the dose, the beam was shifted away from the original field to the first subfield until the dose cloud disappeared. The lateral field was copied as the second subfield. Again, using MLCs for blocking, the dose to the second subfield was $2 \%$ to $4 \%$ lower than the dose blocked in the first subfield, and the beam weight was shifted. Subfields with Monitor Units (MU) > 4 always used. Forward planning based on the 3D dose distribution and on dose-volume histograms (DVHs) in order to reach the best homogeneity of the dose distribution can optimize the MLC positions and beam weighting. If the maximum dose was over $107 \%$ of the prescribed dose, the medial field was copied again as the third subfield. The MLCs were not allowed to block within $1 \mathrm{~cm}$ of the reference point. Figure (1) shows the BEVs for two open beams and two subfields. Figure (2) shows the hot areas when using only two open beams before adding segments and these hot areas were removed after adding three subfields (two subfields medial and one lateral sub field).

\section{Dosimetric evaluation}

\section{Treatment planning (TP) evaluation tools}

There are many tools for qualitative and quantitative evaluation of the TPs.

The visual slice-by slice review of the treatment plans using isodose lines distribution can be used as a qualitative evaluation for the treatment plans where the qualitative evaluation is important to identify the locations of the hot and cold areas in the treatment plans. The quantitative evaluation included the maximum, minimum, mean doses. Dose Volume Histogram (DVH) was generated to evaluate the dose to the different structures in treatment plans. For PTV, the parameters, Dmax, Dmean (the maximum and mean doses in the PTV), V95 \%,V105 \% and V107 \% (the 
percentage of volumes receiving at least 95,105 and $107 \%$ of the prescribed dose) were used for plan evaluation. For OARs, the mean and maximum doses, V20Gy, V5Gy for ipsilateral lung, were used for the treatment plan evaluation. V30,V25,V5,V20, Dmean for heart and Dmax, Dmean, V2,V1.2,V0.4 for contralateral breast, were used for the treatment plan evaluation.

\section{Plan quality}

Dose volume histogram

All plans were optimized to ensure that $95 \%$ of the volume of PTV received $95 \%$ of the prescribed dose of $40 \mathrm{~Gy}$.

\section{Dose homogeneity index}

Some other indices are required for evaluating the dose distribution within the target and also for assessing the healthy tissue doses. The DHI was used for the evaluating recovered dose distribution within the target dose, where lower DHI defines a target dose with a little change. A DVH was used in the calculation of the DHI.

The homogeneity index was defined using many formulas [19]:

$$
D H I=\frac{D_{2}-D_{98}}{D_{\text {prescription }}} \times 100
$$

Where D98 represents the dose to the $98 \%$ of the volume (considered to be the minimum dose) D2 is the dose to the $2 \%$ of the target volume (considered to be the maximum dose).

\section{The conformity index}

Several different conformity indices have been reported to describe the conformity of the prescription isodose to the target volume. The PITV recommended in the Radiation Therapy Oncology Group (RTOG) radiosurgery guidelines is probably the most frequently quoted [20]. Equation 2 defined the PITV as the ratio of the prescription isodose volume (RI) to the target volume size (TV). The RTOG guidelines define a ratio of 1.0-2.0 as per protocol and ratios in the range of $0.9-1.0$ or $2.0-2.5$ as minor variations.

$$
\mathrm{PITV}=\frac{\mathbf{V}_{\mathrm{RI}}}{\mathrm{TV}}
$$

\section{Results \\ Dose Volume Histogram \\ PTV}

The dose volume evaluation for the target with this technique was performed. All patients were optimized to ensure that $95 \%$ of the volume of the PTV received $95 \%$ of the prescribed dose. The data for all patients are presented in Table (1).
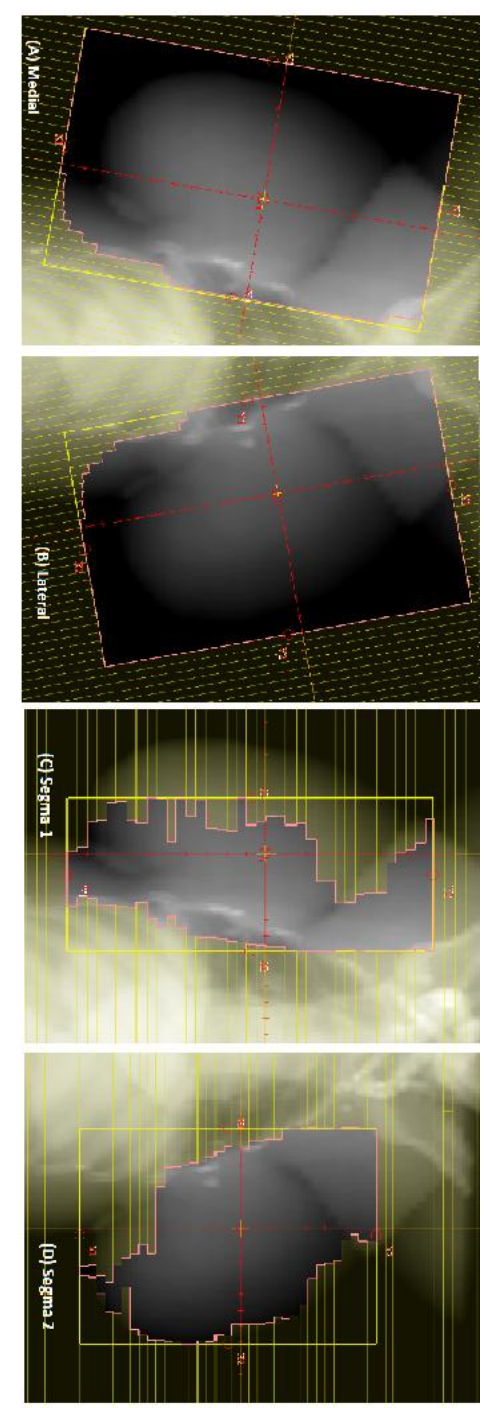

Figure (1) the DRRs for one of ten breast cancer patient $s$ with two open beams and two segments beams 


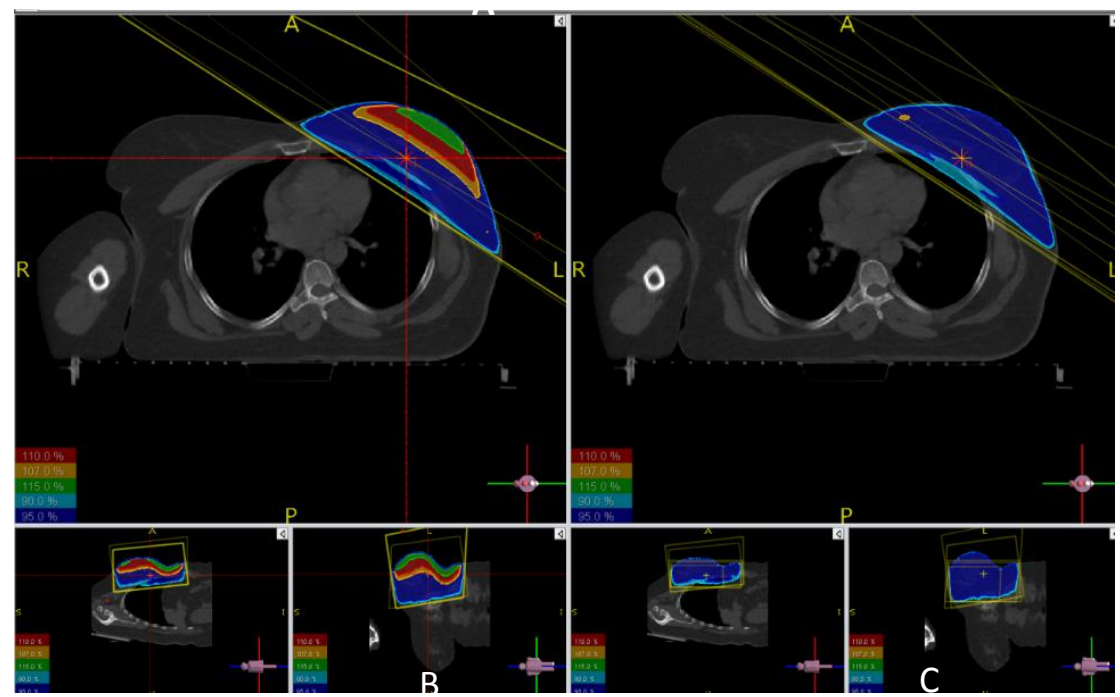

Figure (2) a) Transverse slice , b) Sagital, c) Cronal for two open beams without segment fields d) Transverse slice e) Sagital, f) Cronal for two open beams and three segment fields

Table (1): the Maximum, V95, V105, V107, mean dose and dose homogeneity for the FIF plan of ten patients

\begin{tabular}{|c|c|c|c|c|c|}
\hline $\begin{array}{l}\text { Patient } \\
\text { number }\end{array}$ & $\begin{array}{c}\text { Maximu } \\
\text { m dose }\end{array}$ & $\begin{array}{c}\text { Mean } \\
\text { dose }\end{array}$ & $\begin{array}{l}\text { V9 } \\
5 \%\end{array}$ & $\begin{array}{l}\text { V1 } \\
05\end{array}$ & $\begin{array}{c}\mathrm{DH} \\
\mathrm{I}\end{array}$ \\
\hline 1 & 106.7 & 99.7 & $\begin{array}{c}96 . \\
1\end{array}$ & 0.4 & $\begin{array}{c}0.2 \\
6\end{array}$ \\
\hline 2 & 106.6 & 99.7 & $\begin{array}{c}96 . \\
5\end{array}$ & & $\begin{array}{c}0.2 \\
6\end{array}$ \\
\hline 3 & 107.3 & 99.2 & $\begin{array}{c}95 . \\
8\end{array}$ & 0.7 & $\begin{array}{c}0.2 \\
5\end{array}$ \\
\hline 4 & 107.9 & 99.6 & 96 & 2.3 & $\begin{array}{c}0.2 \\
6\end{array}$ \\
\hline 5 & 107.3 & 99.7 & 95 & 0.2 & $\begin{array}{c}0.2 \\
8\end{array}$ \\
\hline 6 & 107.4 & 101 & $\begin{array}{c}95 . \\
1\end{array}$ & 0.4 & $\begin{array}{c}0.3 \\
4\end{array}$ \\
\hline 7 & 106.1 & 100.8 & 95 & 4.4 & $\begin{array}{c}0.3 \\
2\end{array}$ \\
\hline 8 & 107.6 & 100.3 & $\begin{array}{c}95 . \\
1\end{array}$ & 0.5 & $\begin{array}{c}0.3 \\
3\end{array}$ \\
\hline 9 & 106.8 & 100.8 & $\begin{array}{c}96 . \\
2\end{array}$ & 4.5 & $\begin{array}{c}0.3 \\
2\end{array}$ \\
\hline 10 & 107.2 & 100.6 & $\begin{array}{c}95 . \\
1\end{array}$ & 3.9 & $\begin{array}{c}0.3 \\
2\end{array}$ \\
\hline $\begin{array}{c}\text { Mean } \pm \\
\text { SD }\end{array}$ & $\begin{array}{l}107.09 \\
\pm 0.53\end{array}$ & $\begin{array}{c}100.14 \\
\pm 0.63\end{array}$ & $\begin{array}{c}95 . \\
59 \\
\pm 0 . \\
59\end{array}$ & $\begin{array}{c}1.9 \\
\pm 1 \\
87\end{array}$ & $\begin{array}{c}0.2 \\
9 \\
\pm 0 . \\
04\end{array}$ \\
\hline
\end{tabular}

\section{For OARs}

Table (2) shows mean and standard deviation $( \pm \mathrm{SD})$ of organs at risk (ipsilateral lung, heart, contralateral breast). The dose volume histogram for PTV and OARs is shown in Figure (3).

Table (2): Mean and Standard Deviation $( \pm \mathrm{SD})$ of Organs at Risk dose parameters for FIF plan

\begin{tabular}{lll}
\hline Structures & $\begin{array}{l}\text { Paramet } \\
\text { ers }\end{array}$ & $\begin{array}{l}\text { FIF (Mean } \pm \text { SD } \\
(\%))\end{array}$ \\
\hline Ipsilateral lung & D mean & $6.18 \pm 4.33$ \\
& D max & $98.22 \pm 4.27$ \\
& V20Gy & $7.73 \pm 5.83$ \\
& V5Gy & $9.9 \pm 6.58$ \\
Heart & D mean & $3.2 \pm 1.64$ \\
& V30Gy & $1.74 \pm 1.03$ \\
& V25Gy & $2.58 \pm 1.51$ \\
& V5Gy & $4.52 \pm 2.54$ \\
& V20Gy & $3.46 \pm 1.97$ \\
cont lateral breast & D mean & $0.51 \pm 0.03$ \\
& & \\
(CC) & Dmax & $49 . .79 \pm 89.31$ \\
& V2Gy & $0 \pm .0$ \\
& V1.2Gy & $0.02 \pm 0.42$ \\
& V0.4Gy & $0.21 \pm 0.24$ \\
\hline
\end{tabular}

Arab J. Nucl. Sci. \& Applic. Vol. 51, No.4 (2018) 


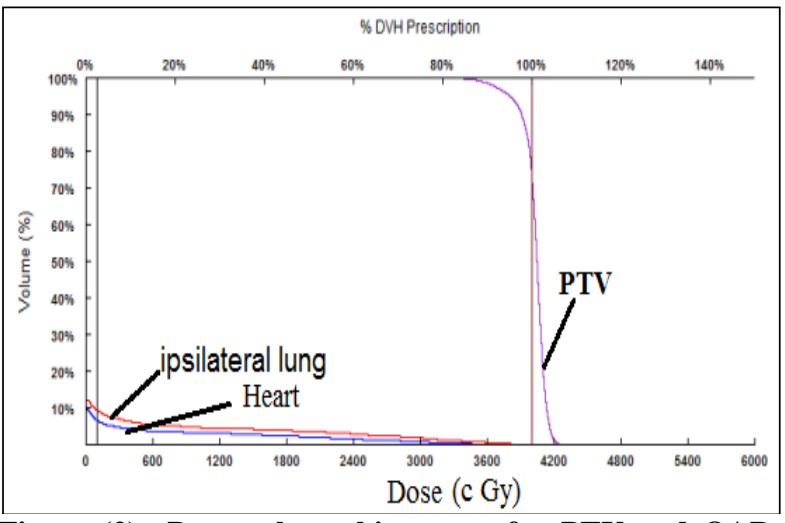

Figure (2) Dose volume histogram for PTV and OARs (ipsilateral lung, and the heart)

\section{Conformity index}

The mean value of PITV for all patients was 1.29 with standard deviation 0.1. Figure (3) summarizes the PITV conformity index for all patients.

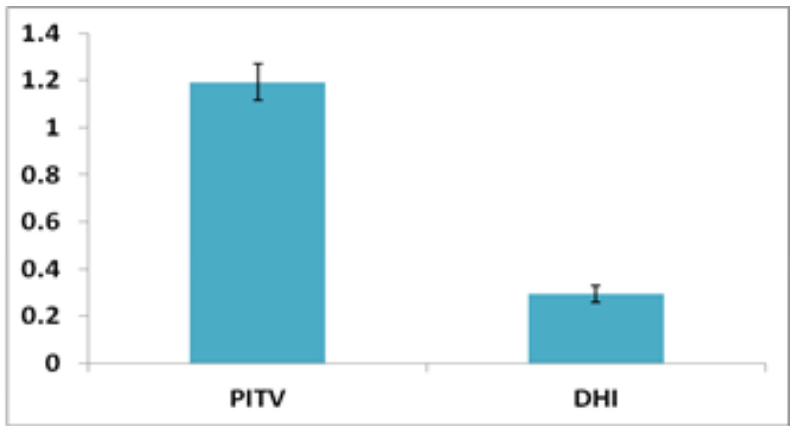

Figure (3): The mean and standard deviation values of PITV for FIF plan of all patients

\section{Discussion}

It has been suggested that poor cosmesis and other late adverse effects are related to the dose inhomogeneity, which is associated with a number of factors, including the irregular shape and large size of the breast [20-21]. The PTV coverage was acceptable if $95 \%$ of the volume were covered by $95 \%$ of the prescribed doses. This was comparable to PTV coverage achieved by the FIF plan. Also, breast reduced the volumes receiving doses over the prescription dose in irradiated volumes and improved the dose homogeneity of PTV. The FIF plan did not only deliver the dose to the intended target, but also spared the doses to critical structure as shown in Table (2). The doses received to all organs were much less than the tolerance dose for all organs, because of the use of MLCs instead of physical wedges which reduced scatter dose to the contralateral breast and other parts of the body [22].
According to RTOG guidelines, ranges of conformity index values have been defined to determine the quality of conformation, because a value of 1 is rarely obtained.

If the conformity index is situated between 1 and 2 , the treatment is considered to comply with the treatment plan. As shown in Figure (3), all the values were close to 1 where the mean values was1.19 \pm 0.1 . So the FIF plan improved the conformity for PTV [13].

The FIF plan was performed using two open tangential fields and two or three subfields were added to open beams with identical gantry angles. It may be stated that MS-CRT (FIF plan) is not true IMRT because only two or three intensity levels per beam are used [8]. This small number of the treatment fields shows some advantages to the FIF technique in comparison with the patients that received IMRT which requires much more number of segments. The small number of segments will make small low-dose areas, less monitor units and short treatment time. This is an important because low-dose areas are suspected to induce secondary cancer as late toxicity and smaller number of monitor units implicates less scattered radiation $[23,24]$. Additional disadvantages of inverseplanned IMRT are that this technique needs more expensive devices, prolonged treatment-planning times, longer treatment-delivery times, and complicated pretreatment quality assurance (QA) procedures [25].

Recently, investigations of hypo fractionated radiotherapy for whole breast radiotherapy have increased [26]. Because that technique always uses a higher prescription dose per fraction compared with conventional fraction radiotherapy, the dose in homogeneities in the irradiated volumes strongly influence radiation-induced late toxicities. Therefore, the volumes of the treated breast and/or the surrounding normal tissues receiving more than the prescribed dose must be decreased to reduce late toxicities after radiotherapy.

\section{Conclusion}

FIF technique for large breast irradiation after breast conserving surgery enables an increase in the dose homogeneity in the PTV. Another benefit of the FIF plan is the disappearance of the scatter dose, which was more prominent in the wedge plan, resulting in fewer contralateral breast doses, possibly causing a reduction in secondary 
malignancy in contralateral breasts. In addition, the FIF plan reduces the doses received by other OARs.

\section{Acknowledgments}

I would like to express my profound gratitude and sincere appreciation to Prof. Dr. Biomy AuudAllah Tartor and Prof. Dr. Amal Ahmed Fouad for their inspiration and indispensable guidance.

\section{References}

1- Fisher,Anderson S;Bryant Jand et al. "Twenty-Year Follow-up of a Randomized TrialComparing Total Mastectomy, Lumpectomy, andLumpectomy Plus Irradiation for the Treatment of Invasive Breast Cancer." N Englj Med 347 (2002): 1233-1241.

2- S, Sautter-Bih, Sedlmaye F, and Budach W and et al, "One life saved by four prevented recurrences?" Strahlenther Onkol 188 (2012): 461-463.

3- Bentel , Marks LB;Whiddon CS and et al. "Acute and late morbidity of using a breast positioning ring in women with large/pendulous breasts." Radiotherap Oncol 50 (1999): 277-81.

4- Das, Shikama N; Cheng CW and et al. "Choice of beam energy and dosimetricimplications for radiation treatment in a subpopulation of women with largebreasts in the United States and Japan." Med Dosimet 31 (2006): 216-23.

5- Tanaka, Ito M;Takahiro Y;amaguchi T and et al,' High Tangent Radiation Therapy With Field-inFieldTechnique for Breast Cancer Breast Cancer: ". Basic and Clinical Research 11(2017):1-5.

6- Onal ,Aydan Sonmez A; Arslan G and et al. "Dosimetric comparison of the field-in-field technique and tangential wedged beams forbreast irradiation.” Jpn J Radiol 30 (2012): 218-226.

7- Gulybán, Kovács P; Sebestyén $\mathrm{S}$ and et al. "Multisegmented Tangential Breast Fields: a Rational Way." Strahlentherapie, 2008: 262-9.

8- Yavas, Yavas C and Acar H. "Dosimetric comparison of whole breastradiotherapy using field in field and conformalradiotherapy techniques in early stage breast cancer." Iran. J. Radiat. Res 10 (2012): 131-138.

9- Hidekazu Tanaka, Hayash S ; Kajiura Yand et al. "Evaluation of the field-in-field technique with lung blocksfor breast tangential radiotherapy." Nagoya J. Med 77 (2015): 339-345.

10- Sasaoka andM Futami. "Dosimetric evaluation of whole breast radiotherapy usingfield-in-field technique in early-stage breast cancer." Int J Clin Oncol 16 (2011): 250-256.

11- TANAKA, Shinya HAYASHI $\mathrm{S}$ and HOSHI H. "Determination of the optimal method for the fieldin-field techniquein breast tangential radiotherapy." Journal of Radiation Research 55 (2014): 769-773.
12- Hany Ammar, Mohammed Elywa, Amal Halim and Shaimaa Elnabawe, ** A Comparative Dosimetric Study on Three-Dimensional Conformal Radiation Treatment and Multisegmented Conformal Radiation Treatment for Breast Cancer', World Journal of Medical Sciences 15 (1): 48-57, 2018

13- Nihe, Mitsumori Ml; Ishigak Tand et al. "Determination of Optimal Radiation Energy for Different Breast Sizes Using CT-simulatior in Tangential Breast Irradiation." Breast Cancer, 2000: 231-236

14- Wu, Mohan R;Morris M and et al. "Simultaneous integrated boost intensity modulated Dosimetric results." Int J Radiat Oncol Biol Phys,10( 2003): 573-585.

15- Neal, Torr M;Helyer S and et aL. "Correlation of breast dose heterogeneity with breast size using 3D CT planning anddose-volume histograms." Radiother Oncol, 1995: 210-8.

16- Donovan,Bleackley NJ; Evans PM and et al. "Doseposition and dose-volume histogram analysis of standard wedged and intensity modulated treatments in breast radiotherapy." $\mathrm{Br} \mathrm{J}$ Radiol, 2002: 967-73.

17- Bhatnagar, Brandner E; Sonnik D and et al. "Intensity modulated radiation therapy (IMRT)reduces the dose to the contralateral breast when compared toconventional tangential fields for primary breast irradiation." Breast Cancer Res Treat. 96 (2006): 41 -

18- Kataria, T., Sharma, K., Subramani, V., Karrthick, K. P., \& Bisht, S. S. (2012). Homogeneity Index: An objective tool for assessment of conformal radiation treatments. Journal of Medical Physics / Association of Medical Physicists of India, 37(4), 207-213.

19- FONTENOT,LEE A K and WAYNE D. "RISK OF SECONDARY MILIGNANT NEOPLASMS FROM PROTON THERAPY ANDINTENSITYMODULATED X-RAY THERAPY FOR EARLYSTAGE PROSTATE CANCER.” Int J 74 (2009): 616-622.

20- Quasi-IMAT Technique and Secondary Cancer Riskin Prostate Cancer." Strahlenther Onkol 183 (2009): 248-53.

21- Mayo, Urie MM; Fitzgerald TJ. "Hybrid IMRT plans-concurrently treating conventional and IMRT beams for improved breast irradiation and reduced planning time.” Int J 61 (2005): 922-932.

22- Whelan , MacKenzie R;Julian J et al. "Randomized trial of breast irradiation schedules after lumpectomy for women with lymph node-negative breast cancer." J Natl Cancer Inst ,94 (2002): 114350.

23- Haviland, Owen JR; Dewar JA; Agrawal RK and et al. "The UK Standardisation of Breast Radiotherapy (START) trials of radiotherapy hypofractionation for treatment of early breast

Arab J. Nucl. Sci. \& Applic. Vol. 51, No.4 (2018) 
cancer: 10-year follow-up results of two randomised controlled trials." Lancet Oncol 14 (2013): 1086-94.

24- Mayo, Urie MM; Fitzgerald TJ. "Hybrid IMRT plans - concurrently treating conventional and IMRT beams for improved breast irradiation and reduced planning time." Int J 61 (2005): 922-932.

25- Whelan , MacKenzie R;Julian J et al. "Randomized trial of breast irradiation schedules after lumpectomy for women with lymph node-negative breast cancer." J Natl Cancer Inst ,94 (2002): 114350

26- Haviland, Owen JR; Dewar JA; Agrawal RK and et al. "The UK Standardisation of Breast Radiotherapy (START) trials of radiotherapy hypofractionation for treatment of early breast cancer: 10-year follow-up results of two randomised controlled trials." Lancet Oncol 14 (2013): 1086-94. 